\title{
One small satellite, so many light curves: Examples of $\delta$ Scuti asteroseismology from the MOST space mission ${ }^{1}$
}

\author{
Jaymie M. Matthews
}

Department of Physics \& Astronomy, University of British Columbia, Vancouver, V6T 1Z1, Canada

\begin{abstract}
The skies are alive with the sound of music. The symphonies of $\delta$ Scuti stars, both postand pre-main sequence, offer more exciting potential for asteroseismology than ever before. Continuous precise light curves of $\delta$ Scuti stars obtained by the MOST (Microvariability \& Oscillations of STars) space mission offer rich eigenspectra and accurate relative mode amplitudes to test models of stellar structure and nonlinear pulsation dynamics. Many of these $\delta$ Scuti pulsators have been discovered among the MOST Guide Star sample. One of them, HD 209775, exhibits more than 80 frequencies, rivalling FG Vir in its richness. The observed amplitude distribution is a test of theoretical mode growth rates and the histogram of frequency spacings places meaningful constraints on the stellar structure and evolutionary phase. MOST has also discovered at least two "hybrid" pulsators, simultaneously exhibiting both $\delta$ Scuti p-modes and $\gamma$ Doradus g-modes, doubling (or tripling) the number of known hybrids. MOST has also been used to target pre-main sequence pulsators (like those in the cluster NGC 2264), performing 'ultrasound' of stellar embryos based on the acoustic oscillations.
\end{abstract}

\section{Austrianasteroseismology}

"The Sound of Music" (Hollywood musical film version) is almost unheard of in Austria. ${ }^{2}$ But the sound of music from stars resonates clearly with Austrian astronomers like Prof. Michel Breger, whose "recording studio" (FG Virginis Records?) has been one of the most successful multi-site photometric networks in history.

It is fitting that this workshop in his honour on the future of asteroseismology should come at a time when space-based missions like MOST, WIRE and COROT are poised to test and extend the ideas about $\delta$ Scuti pulsation that Michel Breger and his team have explored as pioneers from the surface of the Earth. It is equally fitting that the scientific productivity of the Canadian MOST satellite reported in this workshop has been enhanced significantly by the addition of the Vienna ground station to its communications network and by the contributions of Prof. Werner Weiss and his team.

\section{MOST}

MOST (Microvariability \& Oscillations of STars) is a Canadian Space Agency mission (Walker et al. 2003; Matthews et al. 2004) which was originally developed solely for asteroseismology of Sun-like stars, pulsating magnetic CP (rapidly oscillating $A p=r o A p$ ) stars and WolfRayet stars, through ultra-precise high-duty-cycle photometry. Since its launch in June 2003,

\footnotetext{
${ }^{1}$ Based on data from the MOST satellite, a Canadian Space Agency mission, jointly operated by Dynacon Inc., the University of Toronto Institute for Aerospace Studies and the University of British Columbia, with the assistance of the University of Vienna.

2 ... and considered by most Austrians to be a cliché of the same magnitude as Canadians would consider the image of a Mountie riding a polar bear chasing a hockey puck into an igloo while eating a doughnut, eh. Well, okay, I admit, that's actually a pretty genuine image of Canada.
} 
the MOST scientific mission has broadened to include exoplanet search and exploration (e.g., Rowe et al. 2006) including the search for exoplanets of Earth size and mass (Croll et al. 2007; Miller-Ricci et al. 2007). Its capabilities have also broadened to enable precise photometry of the guide stars used for satellite attitude control, resulting to date in about 560 light curves of 480 different stars. This has led to the discovery of $\delta$ Scuti, $\gamma$ Doradus and hybrid pulsators, as well as to $g$-modes among Be supergiants, and nonradial $p$-modes in red giants (Barban et al. 2007; Kallinger et al. 2007)

MOST is a microsatellite ( $54 \mathrm{~kg}$ in mass) housing a CCD photometer fed by a $15-\mathrm{cm}$ Maksutov telescope with a custom broadband optical filter $(350-750 \mathrm{~nm})$. Its polar Sunsynchronous orbit allows it to monitor stars within a near-equatorial Continuous Viewing Zone (CVZ) $54^{\circ}$ wide continuously for up to two months. It is now also possible to monitor stars outside the CVZ with interruptions during each of the 101.4-min satellite orbits, giving very thorough coverage of stellar variability with timescales of a few hours. Point-to-point photometric precision ranges from about $100 \mathrm{ppm}$ for the brightest targets (in Fabry Imaging mode) to a few mmag for targets as faint as $V \sim 11$.

\section{The oscillations of a $\delta$ Scuti star: An Instability Strip tease}

One of the challenges of $\delta$ Scuti asteroseismology is that the theoretical eigenfrequency spectra of the stellar models are so richly populated that it is difficult to find a unique match to the observed frequencies without independent mode identifications. As someone remarked at this workshop, "there is no simple pattern" to recognize in the low-degree, low-overtone pulsation frequencies of a $\delta$ Scuti star - no asymptotic comb of modes that stands out in the high-overtone oscillations of the Sun, $\alpha$ Cen A and B, and roAp stars like HD $24712=$ HR 1217.

But if you detect enough frequencies in a $\delta$ Scuti pulsator, there may indeed be a pattern lurking to be found. A case in point is one of the variable stars in the MOST Guide Star sample: HD 209775.

One of the richest $\delta$ Scuti stars ever

HD $209775(V=7.6)$ is an F0 star which was recognized as a $\delta$ Scuti variable by Henry \& Henry (2000) based on about 10 hours of ground-based photometry. The star was being tested as a possible photometric comparison for the transiting exoplanet system HD 209458, but its pulsational variability makes it unsuitable for this role (see also Henry 2000). Xu et al. (2002) included HD 209775 in their study of the red edge of the instability strip. They derive from Strömgren photometry (Rodriguez et al. 2000) the star's luminosity to be $\log L / L_{\odot} \simeq 0.749$ and its effective temperature to be $T_{\text {eff }} \simeq 7490 \mathrm{~K}$. T

HD 209775 may not have been a suitable comparison star for HD 209458, but it served perfectly as one of five guide stars for MOST monitoring of the transiting exoplanet system, both during a trial run of 14 days during $1-15$ September 2004 and a more extensive run of 44 days during 2 August - 15 September 2005. The 2005 run consisted of more than 369,000 individual exposures sampled every 10 seconds, covering over 1050 hours with only one gap of about 7 hours $-99.4 \%$ duty cycle. Details of the 2004 observations of HD 209458 are described by Rowe et al. (2006) and the 2005 data by Rowe et al. (2007, in preparation).

The star was immediately recognizable as a multi-periodic $\delta$ Scuti variable, even in the raw data. Even the "raw" MOST Guide Star photometry undergoes a first-order background removal on board the satellite. For the 2005 run, the stray light due to scattered Earthshine was very high during the first three weeks, and to be conservative, we removed about $25 \%$ of the data during each MOST orbit. For the final three weeks, the stray light levels were so modest that no data removal was deemed necessary. The overall result is a light curve with a net duty cycle of $84 \%$. A long-term trend apparent in the other four guide stars in 
the field was removed from the photometry with a second-order polynomial fit, and the data were binned to a sampling rate of 2 minutes. Figure 1 shows this preliminary reduction of the 2005 light curve.

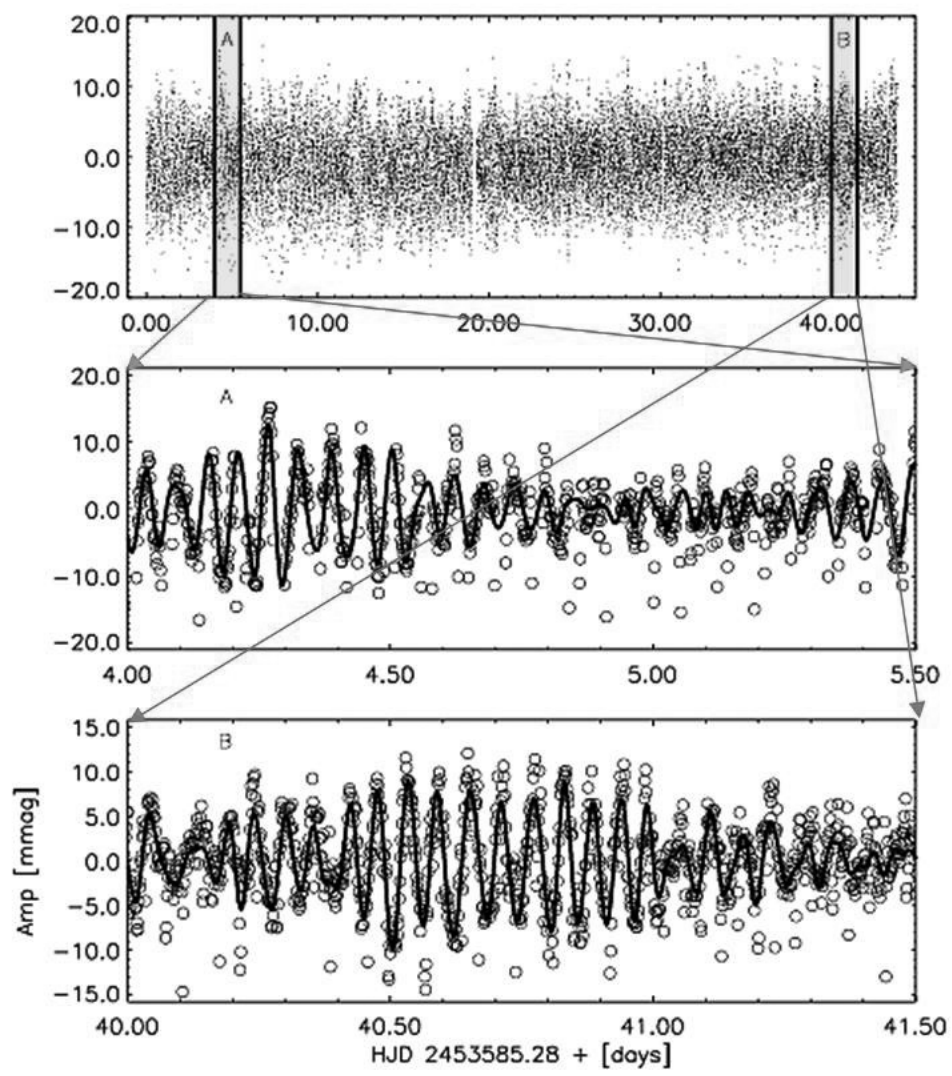

Figure 1: MOST 2005 light curve of HD 209775. Top panel: All 44 days of photometry, binned at a sampling rate of once every $2 \mathrm{~min}$. The $y$-axis is in units of relative variation from the mean, with brightness increasing upwards. Lower panels: Subsets of 1.5 days each, where the solid curves trace the multi-frequency fits to the data. 
The reduced photometry was searched for periodicities through a discrete Fourier transform, nonlinear least squares fitting (with algorithms similar to Period04, developed under M. Breger's supervision in Vienna) and bootstrapping techniques. The Fourier amplitude spectrum of the data is plotted in Fig. 2 and shows the abundance of frequencies in the range $0-35 \mathrm{c} / \mathrm{d}$. There are 88 frequencies present in the $\delta$ Scuti $p$-mode range, whose peaks have an amplitude $\mathrm{S} / \mathrm{N}$ greater than 3.6. The largest amplitude is only around 2 millimag.

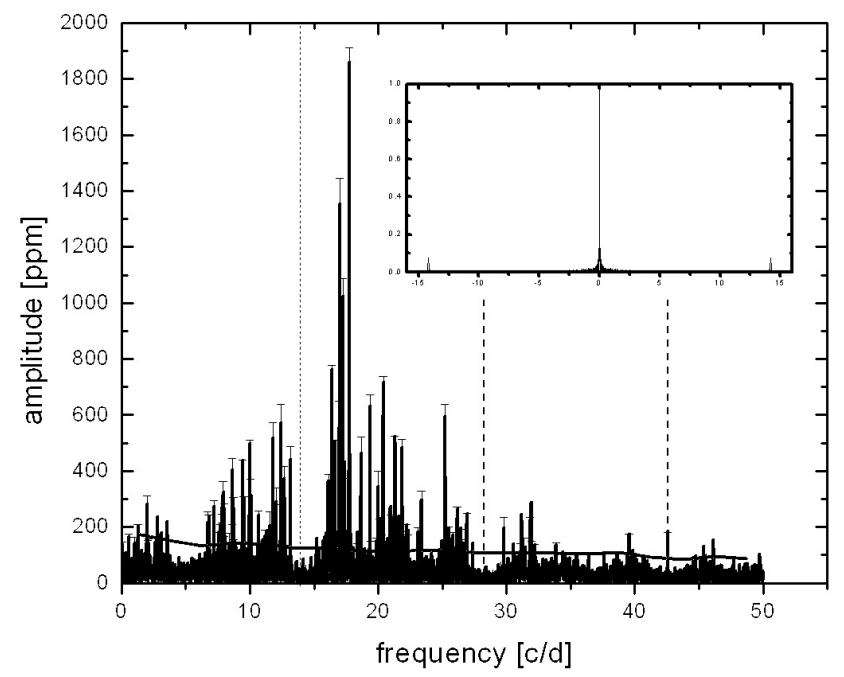

Figure 2: Fourier amplitude spectrum of the HD 209775 time series. The vertical dashed lines mark the orbital frequency of the MOST satellite and its first two harmonics. The inset is the spectral window of the time series, where two small sidelobe peaks are present, spaced by $14.2 \mathrm{c} / \mathrm{d}$ (the orbital frequency of MOST).

Modelling growth rates and frequency separations

HD 209775 joins FG Vir (Breger et al. 2005) as one of the richest multi-periodic $\delta$ Scuti stars ever observed. One advantage of detecting so many modes and combination frequencies in a single 45-day-long observing run is that it is possible to study the relative mode amplitudes in the envelope of excited peaks to test theories of pulsational growth rates. Indeed, such a rich eigenspectrum from a single observing sequence, prompted Moya, Goupil, Dupret, Michel and Baglin (cf. Matthews et al. 2007, in preparation) to construct models of HD 209775 from two evolutionary codes adapted specifically for asteroseismic studies: CESAM (Morel 1997) and CLES (Scuflaire 2005; see Miglio et al. 2007 for an application of CLES). In Fig. 3, the growth rates $\eta$ for one model from the CESAM grid are compared to the observed range of frequencies in HD 209775. This model has $M / M_{\odot}=1.74, \log T_{\text {eff }}=3.866$ and $\log L / L_{\odot}=1.087$, and of the four models tested, has positive growth rates closest to the low end of the observed frequency range. The other models ranged in mass from 1.55 to $1.80 M_{\odot}$ and $L / L_{\odot}$ from 0.753 to 1.137 . 
The Hipparcos parallax of HD 209775 (10.17 \pm 0.94 mas) and the apparent magnitude of the star indicate a luminosity of $0.85 \pm 0.08 L_{\odot}$, less luminous than the model fit shown above. Note that only four models have been generated in this preliminary analysis and a more complete model grid will be explored.

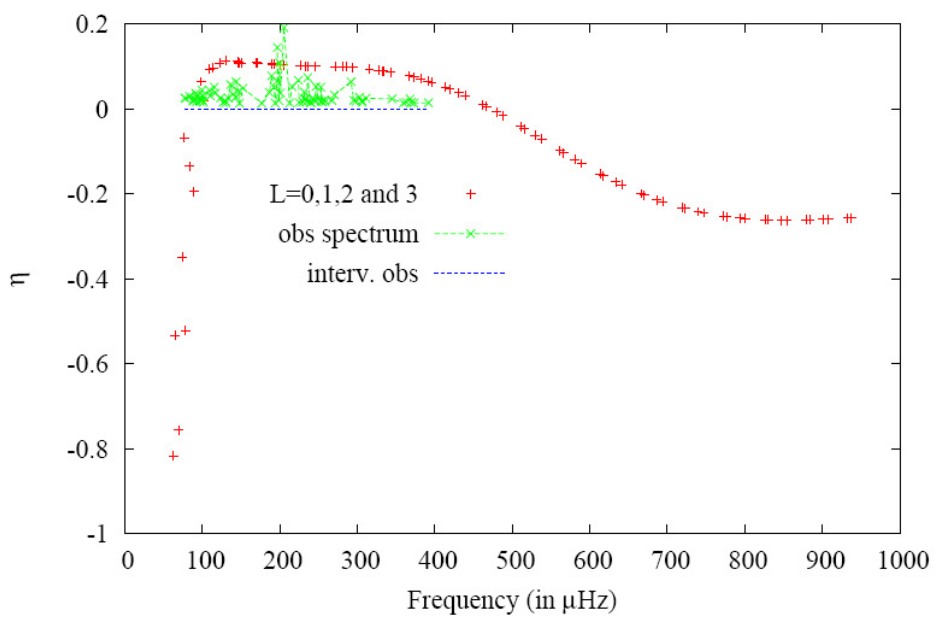

Figure 3: Growth rates of $p$-modes $(\ell=0,1,2$ and 3$)$ as a function of frequency for a CESAM model consistent with the observed parameters (and uncertainties) of HD 209775, compared to the observed frequency spectrum. The model parameters are given in the text.

For a star like HD 209775, with no multicolour photometry nor spectral line profile variability data to identify individual modes, one effective way to compare the observed frequencies to models is through histograms of frequency differences (Breger et al. 1999, Goupil et al. 2000). Moya et al. computed the differences between the mean frequencies identified in the MOST photometry and counted the number of differences in a given interval to plot the number as a function of frequency difference, for different binning intervals (or widths). These can then be compared to theoretical histograms from the stellar model eigenspectra, including and neglecting rotational splitting. The best agreement in the preliminary analysis is shown in Fig. 4.

The histogram method constrains the large frequency separation, which is essentially proportional to the dynamical time of the models. For HD 209775, the best fitting large separation $\Delta \nu$ is around $50 \mu \mathrm{Hz}$, corresponding to $\left(R / R_{\odot}\right)^{3} /\left(M / M_{\odot}\right) \sim 5.6$. Combined with the comparison of the model instability regions to the observed range of excited frequencies, the most luminous models agree best with the data. (More luminous $\delta$ Scuti stars are expected to exhibit more eigenmodes, but the better sampled histograms are not the reason for the better match found for the model shown.)

Rotational splitting must be included to get a good match to the difference histogram with modes only up to $\ell=3$. (There was no published estimate of vsini for this star, so Artie Hatzes obtained for the MOST team a high-resolution spectrum of HD 209775 in September 2005 from the Tautenburg Observatory, yielding a rough estimate of $v \sin i=75 \pm 5 \mathrm{~km} / \mathrm{s}$.) The effects of metallicity have not been included in the preliminary analysis and a more complete investigation is underway. 


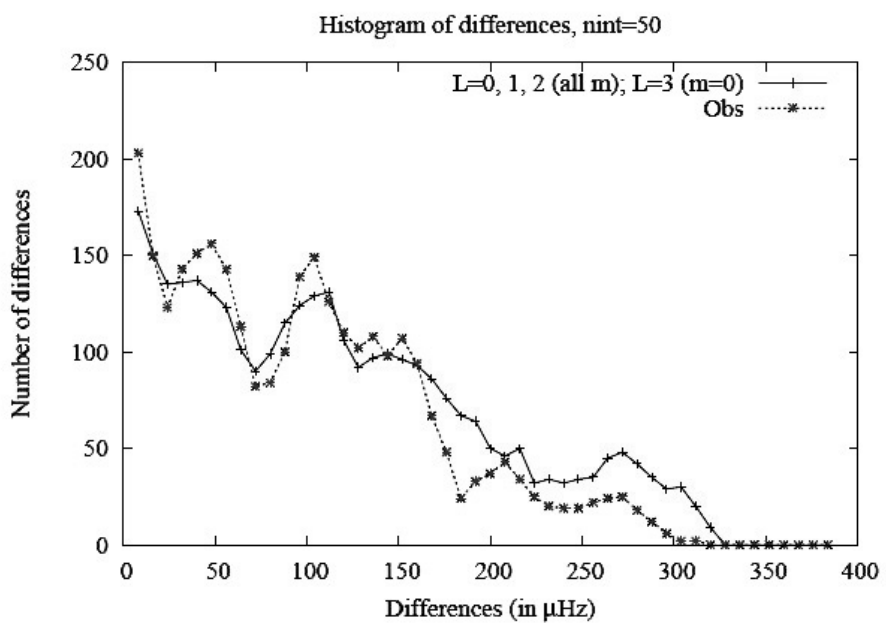

Figure 4: Preliminary comparison of the frequency difference histograms (in $50-\mu \mathrm{Hz}$ bins) of the observed frequencies and the best matching model, which includes rotational splitting but requires only low-degree modes up to $\ell=3$.

\section{Hybrids and embryos}

MOST has discovered two new hybrid pulsators, which exhibit simultaneous oscillations consistent with $\delta$ Scuti $p$-modes and $\gamma$ Doradus g-modes: BD+18 4914 (Rowe et al. 2006a) and HD 114839 (King et al. 2006). Both are Am stars. Only two hybrids were previously known: HD 209295 (Handler \& Shobbrook 2002) and HD 8801 (Henry \& Fekel 2005). The former is a close binary for which the authors argue that the $g$-modes are excited by tidal interaction. The latter is an Am star which is not known to be part of a binary system. The MOST additions to the hybrid sample - if demonstrated to be single stars - may point to the importance of the Am peculiarity to the hybrid pulsation phenomenon. There are other hybrid candidates in the MOST guide star sample and identifying them will help define the dual instability parameter space (Gruberbauer, MSc thesis, in preparation).

The $\delta$ Scuti instability strip covers pre-main-sequence (PMS) evolutionary tracks as well as post-MS phases. MOST has also observed the pulsations of PMS stars, in particular two in the young open cluster NGC 2264: V588 Mon and V589 Mon. In December 2005 - January 2006, MOST monitored these stars and others in the cluster for about 48 days with nearly continuous time coverage. Segments of the PMS light curves are shown in Fig. 5. About 150 significant frequencies were identified in V588 Mon, of which about 90 are combination frequencies; in V589 Mon, $\sim 90$ frequencies and $\sim 40$ combinations. Asteroseismic analyses of the p-mode eigenspectra are underway (Kallinger et al. 2007, in preparation).

Another PMS pulsator observed by MOST is the field star HD 142666, monitored for 11.5 days with a 70\% duty cycle. HD 142666 (Zwintz et al. 2007, in preparation) exhibits pronounced variations like another PMS star UX Ori, attributed to a clumpy disk seen nearly edge-on, as well as $\delta$ Scuti pulsations. 


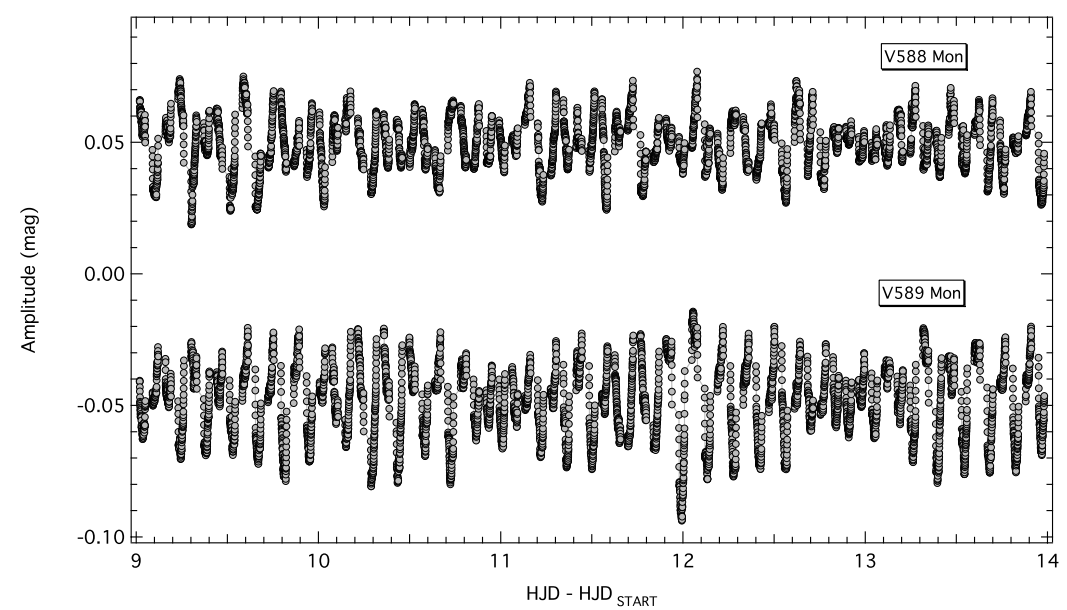

Figure 5: Five-day segments of the MOST light curves of the pulsating PMS stars V588 and V589 Mon in the cluster NGC 2264. The y-axes are in units of relative variation from the mean, with brightness increasing upwards.

\section{On the verge of breakthroughs}

The near future promises (1) additional rich $\delta$ Scuti and hybrid eigenspectra from the ultraprecise long-time-coverage photometry of MOST, WIRE, COROT, and Kepler, plus (2) precise line-profile variability data (and mode identifications) from multi-site high-resolution spectroscopic networks like SONG, and (3) improving models of pulsation with the effects of rotation. This is a combination that $\delta$ Scuti asteroseismologists have awaited for decades, and that Michel Breger and his colleagues have prepared us for with patient and meticulous observations and models over those same decades.

Acknowledgments. Thanks, Mike!

\section{References}

Barban C., Matthews J. M., De Ridder J., et al. 2007, A\&A, in press

Breger M., Pamyatnykh A. A., Pikall H., Garrido R., 1999, A\&A 341, 151

Breger M., Lenz P., Antoci V., et al., 2005, A\&A, 435, 955

Croll B., Matthews J. M., Rowe J. F., et al., 2007, ApJ, in press

Goupil M.-J., Dziembowski W. A., Pamyatnykh A. A., Talon S., 2000, in Breger M., Montgomery M. H., eds, ASP Conf. Ser. Vol. 210, Delta Scuti and Related Stars. Astron. Soc. Pac., San Francisco, p. 267

Handler G., Shobbrook R. R., 2002, MNRAS, 333, 251

Henry G. W., 2000, ApJ, 529, L41

Henry G. W., Fekel F. C., 2005, AJ, 129, 2026

Henry G. W., Henry S. M., 2000, IBVS, 4826 
Kallinger T., Guenther D. G., Weiss W. W., et al., 2007, ApJ, submitted

King H., Matthews J. M., Cameron C., et al., 2006, Comm. Asteroseis., 148, 28

Matthews J. M., Kuschnig R., Guenther D. B., et al., 2004, Nat 430, 51

Matthews J. M., Kuschnig R., Moya A., et al., 2007, in preparation

Miglio A., Montalban J., Dupret M.-A., 2007, MNRAS, 375, L21

Miller-Ricci E., Rowe J. F., Sasselov D., et al., 2007, ApJ, in press

Morel P. 1997, A\&A 124, 597

Rodriguez E., Lopez-Gonzalez M. J., Lopez de Coca P., 2000, A\&AS, 144, 469

Rowe J. F., Matthews J. M., Seager S., et al., 2006, ApJ, 646, 1241

Rowe J. F., Matthews J. M., Cameron C., et al., 2006a, Comm. Asteroseis., 148, 34

Rowe J. F., Matthews J. M., Seager S., et al., 2007b, in preparation

Scuflaire R., 2005, in the 4th COROT/ESTA meeting (Aarhus, Denmark), http://www.astro.up.pt/corot/welcome/meeting

Walker G. A. H., Matthews J., Kuschnig R., et al., 2003, PASP, 115, 1023

Xu Y., Li Z.-P., Deng L.-C., Xiong D.-R., 2002, ChJAA, 2, 448

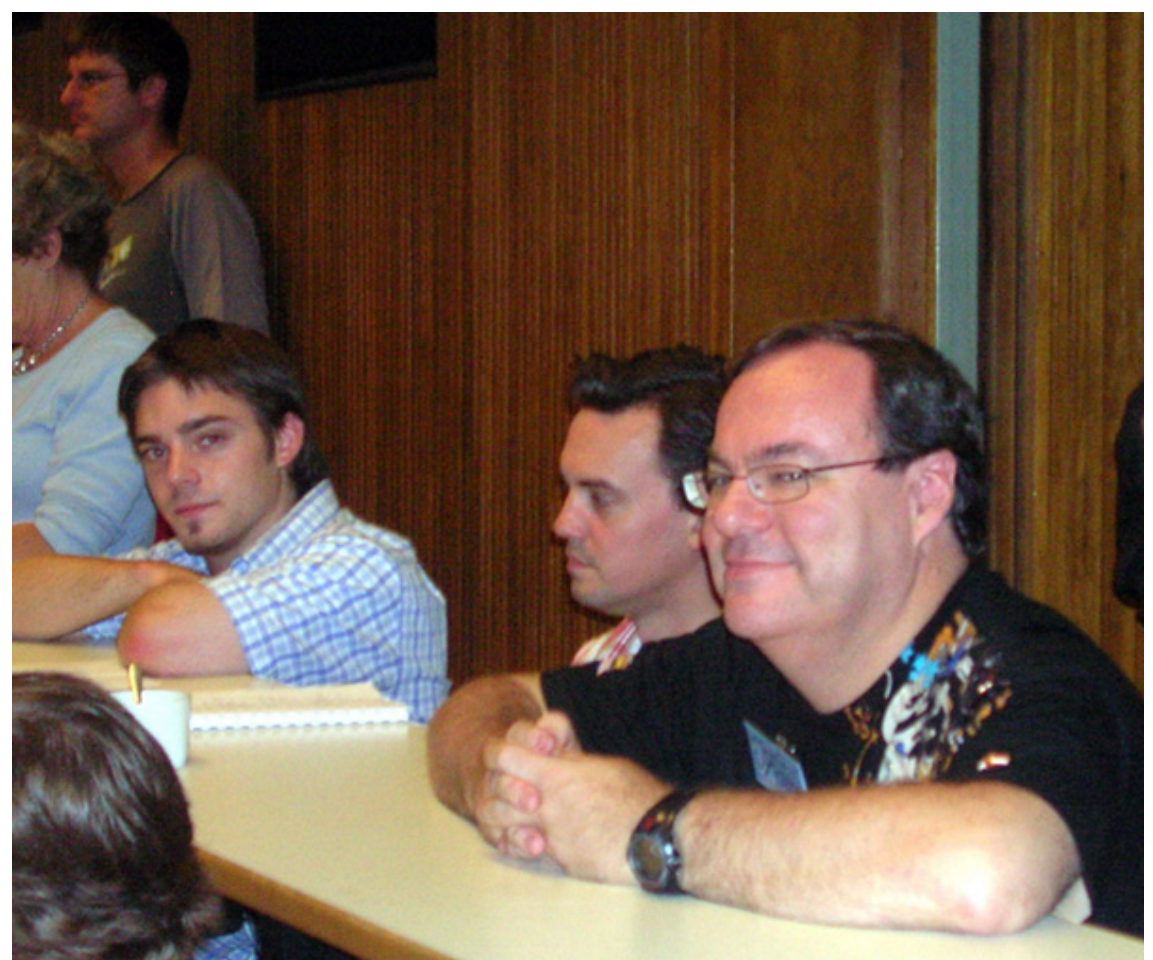

Michael Gruberbauer, Thomas Kallinger and Jaymie Matthews - thinking about science? 